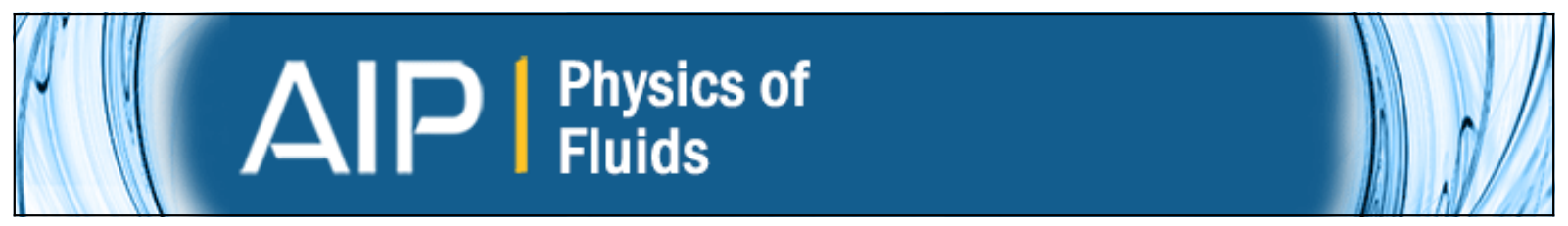

Power-law versus log-law in wall-bounded turbulence: A large-eddy simulation perspective

W. Cheng and R. Samtaney

Citation: Physics of Fluids (1994-present) 26, 011703 (2014); doi: 10.1063/1.4862919

View online: http://dx.doi.org/10.1063/1.4862919

View Table of Contents: http://scitation.aip.org/content/aip/journal/pof2/26/1 ?ver=pdfcov

Published by the AIP Publishing

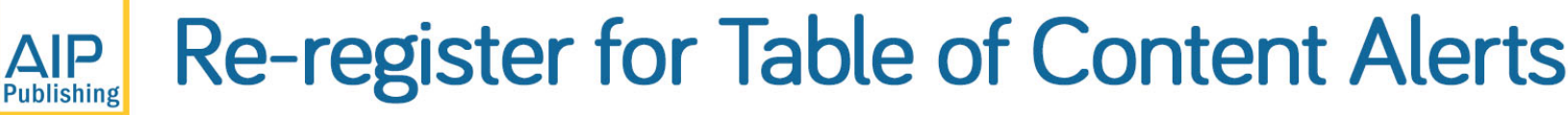




\title{
Power-law versus log-law in wall-bounded turbulence: A large-eddy simulation perspective
}

\author{
W. Chenga) and R. Samtaney \\ Physical Sciences and Engineering Division, KAUST, Thuwal 23955-6900, Saudi Arabia
}

(Received 28 September 2013; accepted 9 January 2014; published online 29 January 2014)

\begin{abstract}
The debate whether the mean streamwise velocity in wall-bounded turbulent flows obeys a log-law or a power-law scaling originated over two decades ago, and continues to ferment in recent years. As experiments and direct numerical simulation can not provide sufficient clues, in this study we present an insight into this debate from a large-eddy simulation (LES) viewpoint. The LES organically combines state-of-the-art models (the stretched-vortex model and inflow rescaling method) with a virtual-wall model derived under different scaling law assumptions (the loglaw or the power-law by George and Castillo ["Zero-pressure-gradient turbulent boundary layer," Appl. Mech. Rev. 50, 689 (1997)]). Comparison of LES results for $\operatorname{Re}_{\theta}$ ranging from $10^{5}$ to $10^{11}$ for zero-pressure-gradient turbulent boundary layer flows are carried out for the mean streamwise velocity, its gradient and its scaled gradient. Our results provide strong evidence that for both sets of modeling assumption (log law or power law), the turbulence gravitates naturally towards the log-law scaling at extremely large Reynolds numbers. @ 2014 AIP Publishing LLC. [http://dx.doi.org/10.1063/1.4862919]
\end{abstract}

Turbulence is ubiquitous in nature. It is characterized by chaotic behavior and stochasticity, instantaneously unpredictable but statistically regular. A key statistical property of wall-bounded turbulent flow is the mean streamwise velocity. The classical interpretation of the mean streamwise velocity is obtained from the overlap assumption following Millikan in 1938, by matching the law of the wall in inner region by Prandtl and the defect law in outer region by von Kármán. This classical explanation results in a logarithmic profile of the mean streamwise velocity in the overlap region and is dubbed the "log law." Mathematically, $U^{+}=\frac{1}{\kappa} \log z^{+}+B$, where $U^{+}$is the mean streamwise velocity component scaled by the friction velocity $u_{\tau}=\sqrt{\tau_{w} / \rho}$, and $\tau_{w}, \rho$ are the wall shear stress and fluid density, respectively. The distance from the wall, $z^{+}$, expressed in "wall units," is the distance scaled by the viscous length $\delta_{v} \equiv v / u_{\tau}$, where $v$ is the fluid viscosity. Although the law for the inner region is well accepted, the debate over the scaling law for outer region is still ongoing. An alternative to the log law is the notion that $U^{+}$has a power law dependence on $z^{+}$in the overlap region. Arguably the three most dominant proponents for a powerlaw behavior may be attributed to the original works by Barenblatt, Chorin, and Prostokishin ${ }^{1,2}$ (BCP law), George and Castillo ${ }^{3,4}$ (GC law), and Zagarola and Smits ${ }^{5}$ (ZS law). In addition, there are other proposed power laws, such as those expressed by Afzal ${ }^{6}$ and McKeon, ${ }^{7}$ but these may be considered as reinterpretations or further developments of the BCP, GC, and ZS power laws.

Each scaling law discussed above is supported by evidence from existing experimental and numerical data. However, evaluations based on experimental and simulation results show that no specific law is valid for the entire overlap region. ${ }^{8}$ Although the power-law scaling arguments do

\footnotetext{
a) Present address, Graduate Aerospace Laboratories, California Institute of Technology. Corresponding email:
} chengw@caltech.edu 
possess a theoretical advantage (a reasonable asymptotic state assumption), a recent comparison between the integral properties of the scaling laws and experimental data indicates superiority of the classic log-law over the power-law scaling in the low Reynolds number regime. ${ }^{9}$ One expects the debate to continue until convincing data at high Reynolds numbers are available. ${ }^{10-12}$ Currently, experiments have achieved relative high Reynolds numbers but it is still not enough to provide a definitive closure to the power-law versus log-law debate. An alternative to experiments and theoretical arguments are computations to address this debate. While Direct Numerical Simulation (DNS) is the most ideal approach for turbulence simulation, DNS capability is restricted by its grid requirement $\left(\mathscr{O}\left(R e^{9 / 4}\right)\right)$ and hence limited to about $R e_{\theta} \sim 10^{4}$ for turbulent boundary layer (TBL) flows. On the other hand, large-eddy simulation (LES), especially those with $\mathscr{O}(1)$ grid dependence on $R e$, can reach extremely high Reynolds number ${ }^{13}$ flows because in that case the overlap layer will almost dominate the near-wall flow. ${ }^{11}$ Our particular emphasis is on the LES of TBL flows with extremely high Reynolds numbers, specifically aimed at shedding more light on the power-law versus log-law debate.

LES of wall-bounded flows generally requires a subgrid-scale (SGS) model and wall model. Among the existing approaches, a framework by Pullin and co-workers, which includes the stretchedvortex SGS model ${ }^{14}$ and a virtual-wall model, has successfully predicted channel flows and TBL flows. ${ }^{13,14}$ In this LES framework, the SGS originates from the ansatz that small scales in turbulence are dominated by stretched-vortices. Among the desirable features of this SGS model are the exclusion of any explicit filtering operations and no empirical constants. The virtual-wall model, combined organically with the SGS model, also does not require any experimental constants, and has unique characteristics when compared with other existing wall models. ${ }^{14}$ Here we begin with this SGS+virtual-wall LES framework to perform our simulations. In establishing the virtual wall model, an assumption of inner scaling is required to get relation between velocity and local wall friction. The original virtual-wall model ${ }^{13,14}$ assumes a classical inner scaling which results in log law. However, if such an assumption is already built into the virtual wall model, one may argue that such LES will depict a bias towards the log-law scaling. To address this concern, we have further developed the virtual-wall model based on a power-law scaling. Here the available choices are one (and possibly more than one) of the aforementioned BCP, GC, and ZS power-laws. Among the three available power-law scalings, the BCP law was eliminated because it does not provide a clear definition of the Reynolds number in the power law for TBL flow. ${ }^{2}$ The choice of the ZS power law is also eliminated because it quickly relapses back to the log law at moderate Reynolds numbers. ${ }^{15}$ The GC law is the most complete formulation ${ }^{9}$ and one that is suitable for implementation in to the virtual-wall model for LES. Therefore, in the present study, we employ the GC power law. We note here that the "asymptotic invariance principle," used in the development of the GC law, suggests a log law for pipe and channel flow, ${ }^{16}$ and a power law for TBL. ${ }^{3}$ This is indeed the rationale for our focus on TBL flows as opposed to channel or pipe flows. Furthermore, we restrict ourselves to the zero-pressure-gradient turbulent boundary layer (ZPGTBL). To reiterate, we employ two flavors of the virtual-wall model within the LES: one based on the assumption of the inner scaling for the log law and one based on the inner scaling for the GC power law. We note that the "constants" in these models (e.g., the Kármán constant, $\kappa$, and others) are not a priori chosen but rather evaluated dynamically during the course of the simulations.

There are three important steps in deriving the virtual wall model: ${ }^{14}$ near-wall filtering, local inner scaling, and velocity integration in overlap region. In the latter two steps, some assumptions based on the classic inner scaling for log law are made in the original virtual-wall model derivation. ${ }^{14}$ To exactly replace the assumption of scaling law, the main idea is to take into account the influence of Reynolds number. In the classic inner scaling for log law, the normalized mean velocity is considered as a function of normalized wall distance, $U^{+}=F\left(z^{+}\right)$. However, the power law is essentially based on the notion of incomplete similarity ${ }^{1}$ which makes $U^{+}$a function of both $z^{+}$and $R e$, where $R e$ is a Reynolds number based on some boundary layer thickness. Following this philosophy, the inner scaling is written as $U^{+}=F\left(z^{+}, R e\right)$. With the assumption of scaling law, one may derive the transport equation of the local wall streamwise velocity gradient $\left.\eta_{w} \equiv(\partial \tilde{u} / \partial z)\right|_{z=0}$ from the filtered momentum equation of streamwise velocity $\tilde{u}$, where the " $"$ denotes the LES filtered variable. To 
derive terms of $\eta_{w}$ from terms of $\tilde{u}$, we differentiate $\tilde{u}$ with respect to $\eta_{w}$ to yield

$$
\frac{\partial \tilde{u}}{\partial \eta_{w}}=\frac{\partial u_{\tau}}{\partial \eta_{w}} F+u_{\tau} \frac{\partial F}{\partial z^{+}} \frac{\partial z^{+}}{\partial \eta_{w}}+u_{\tau} \frac{\partial F}{\partial R e} \frac{\partial R e}{\partial \eta_{w}},
$$

where the first two terms are the same as that in the original wall model and have a simple form after the operation of the wall-adjacent average filter. The last term, which is the influence of $R e$, is an additional term attributed to the incomplete similarity assumption in the power law. With $\beta$ expressing the influence of $R e$, the time evolution ordinary differential equation (ODE) for $\eta_{w}$ is finally obtained as

$$
\begin{aligned}
& \frac{\left.\tilde{u}\right|_{h}}{2 \eta_{w}}(1+\beta) \frac{d \eta_{w}}{d t}+\frac{\left.\partial \widetilde{u u}\right|_{h}}{\partial x}+\frac{\left.\partial \widetilde{u v}\right|_{h}}{\partial y}= \\
& -\left.\frac{1}{h} \widetilde{u w}\right|_{h}-\left.\frac{\partial \widetilde{p}}{\partial x}\right|_{h}+\frac{v}{h}\left(\left.\frac{\partial \tilde{u}}{\partial z}\right|_{h}-\eta_{w}\right) .
\end{aligned}
$$

After solving the local wall friction, a typical power law with the form of $U^{+}=C\left(z^{+}+a^{+}\right)^{\gamma}$ with $a^{+}$a shifted origin, parameters $C$ and $\gamma$ is used to get the local velocity boundary condition. The GC power law can be written as

$$
U^{+} \equiv \frac{U}{u_{\tau}}=C_{i}\left(\frac{z+a}{l^{+}}\right)^{\gamma}
$$

Here the Reynolds number dependent parameters $C_{i}, C_{o}$, and $\gamma$ are empirically fitted from experimental data: ${ }^{3}$

$$
\begin{aligned}
\gamma & =\gamma_{\infty}+\frac{\alpha A}{\left(\ln R e_{c}\right)^{1+\alpha}}, \\
\frac{C_{o}}{C_{i}} & =\frac{C_{o \infty}}{C_{i \infty}} \exp \left[(1+\alpha) A /\left(\ln R e_{c}\right)^{\alpha}\right], \\
\frac{C_{o}}{C_{o \infty}} & =1+c_{1} \exp \left(c_{2} R e_{c}\right),
\end{aligned}
$$

where $R e_{c}$ defined as $\delta_{99} u_{\tau} / \nu$ and $a^{+} \approx-16, \gamma_{\infty}=0.0362, A=2.90, \alpha=0.46, C_{o \infty}=0.897$, $C_{i \infty}=55, c_{1}=0.283, c_{2}=-0.00598$. Assuming that the filtered velocity $\tilde{u}$ follows the GC law, the factor $\beta$ is then evaluated as $\beta=\left.\tilde{u}\right|_{h} \mathscr{F} / 2$ with

$$
\mathscr{F}(R e)=\frac{1}{\gamma}\left(\frac{c_{1} c_{2} R e}{e^{-c_{2} R e}+c_{1}}+(1+\alpha) A \alpha \frac{\ln \left[R e /\left(z^{+}+a^{+}\right)\right]}{(\ln R e)^{2+\alpha}}\right) .
$$

It is found that $\beta$, about two orders of magnitude smaller than unity, decreases with Reynolds number and ranges from 0.05 at $R e_{\theta}=10^{5}$ to 0.01 at $R e_{\theta}=10^{12}$. With the model derived so far, the virtual-wall boundary condition can be implemented; and the ODE Eq. (2) is integrated along with the LES of the main flow. In the implementation of the virtual wall boundary condition, $\tilde{u}$ is computed according to Eq. (3).

An important issue specific to simulating ZPGTBLs with open streamwise boundaries is that of turbulent flow generation at the inflow boundary. The method employed is a rescaling method first developed by Lund et al. ${ }^{17}$ It is noted that Lund rescaling method intrinsically assumes the log-law scaling. This again is an assumption that needs to be relaxed in order to systematically examine the power-law versus log-law issue. With this in mind, we implement two versions to generate the turbulent inflow boundary condition: one obvious choice is the Lund method with log-law scaling, and the other is an extension of this method with scaling parameters chosen to follow the GC power-law. ${ }^{18}$

The code, based on a fractional step fourth-order finite difference method and third order time integration, has been verified extensively against known solutions of the Navier-Stokes equations; and validated against existing ZPGTBL experiments faithfully reproducing experimental mean velocity profiles and turbulent intensities (details in Ref. 19). The convective terms are evaluated explicitly using an energy conservative skew-symmetric form. LES is carried out for cases with a large range of 

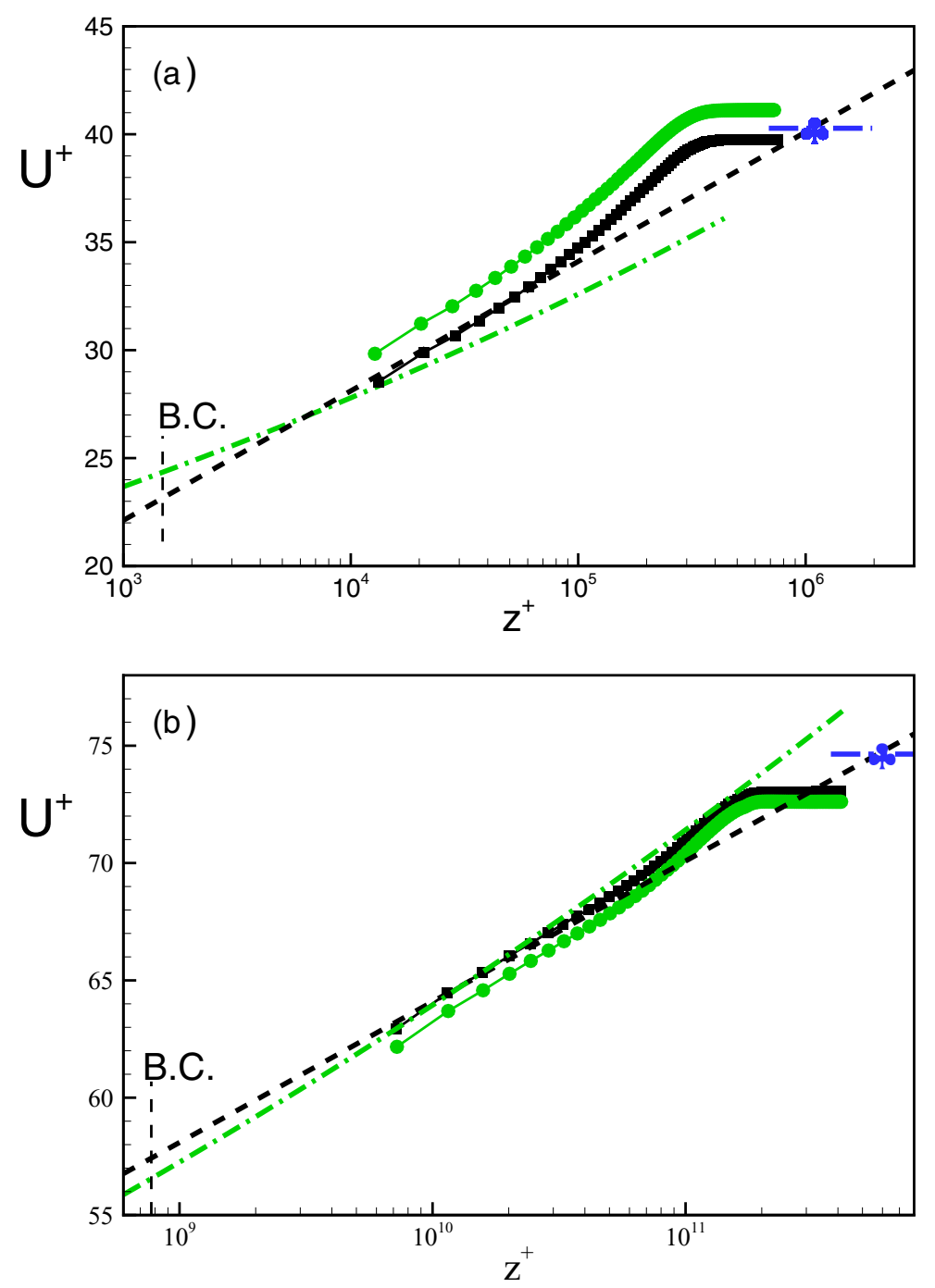

FIG. 1. Mean streamwise velocity profiles. (a) $R e_{\theta}=1.1 \times 10^{6}$; (b) $R e_{\theta}=6.6 \times 10^{11}$. Square symbols: LES with $\log$-law; circle symbols: LES with power-law; dashed line: $\log$-law fit with $\kappa=0.384$ and $B=4.127$; dashed-dotted line: GS law fit; line with symbol \&: theoretically predicted $U_{\infty}^{+},{ }^{20}$ short dashed line with B.C. indicates the approximate location where the virtual wall boundary condition is applied.

Reynolds numbers, from $R e_{0}=10^{5}$ to $R e_{0}=10^{13}$, where $R e_{0}$ is defined based on the $99 \%$ boundary thickness at the inflow $\delta_{0}$. For each Reynolds number the LES is computed assuming either a $\log$ law (denoted as LES-L) or the GC power law (denoted as LES-P) in the virtual wall model and the inflow generation. The simulation domain is $48 \delta_{0} \times 3 \delta_{0} \times 4 \delta_{0}$ box domain discretized with a $512 \times 32 \times 128$ mesh. All data compared following are extracted at about $60 \%$ of the streamwise domain and mean velocity $U^{+}$is averaged temporally and in the spanwise direction.

In Fig. 1, we plot profiles of $U^{+}$for two different Reynolds numbers: one relatively small $R e_{\theta}=1.1 \times 10^{6}$ and the other corresponding to a relatively large Reynolds number of $6.6 \times 10^{11}$. For comparison, the log-law fit and the power-law fit are also shown. A theoretical estimate of the scale free stream velocity $U_{\infty}^{+}$(as the line with "club" symbol), plotted in the same figure, shows that $u_{\tau}$ predicted by both LES-P and LES-L are in good agreement (within a relative error of 3\%) with the "Coles-Fernholz" formula. ${ }^{20}$ It should be noted that for $\operatorname{Re}_{\theta}=1.1 \times 10^{6}$ case, the whole velocity profile in LES-L is offset below that of LES-P. Omitting a detailed explanation, we attribute this offset due to the differences in wall models between LES-L and LES-P at about $z^{+} \approx 1.5 \times 10^{3}$ (denoted in Fig. 1(a) as short dashed line with "B.C.," the approximate location where the virtual 
TABLE I. Relative difference $\eta$ between the velocity gradient obtained from LES and that predicted by the theoretical log-law or power-law scaling.

\begin{tabular}{cccc}
\hline \hline & $\operatorname{Re}_{\theta}$ & Log-law & GC-law \\
\hline LES-L & $1.4 \times 10^{4}$ & $18 \%$ & $20 \%$ \\
& $1.2 \times 10^{5}$ & $14 \%$ & $26 \%$ \\
$1.0 \times 10^{6}$ & $11 \%$ & $24 \%$ \\
& $9.2 \times 10^{6}$ & $9.6 \%$ & $20 \%$ \\
& $8.1 \times 10^{7}$ & $8.1 \%$ & $15 \%$ \\
& $7.8 \times 10^{8}$ & $7.0 \%$ & $6.6 \%$ \\
& $7.2 \times 10^{9}$ & $5.3 \%$ & $5.4 \%$ \\
& $6.9 \times 10^{10}$ & $4.6 \%$ & $13 \%$ \\
LES-P & $6.6 \times 10^{11}$ & $2.8 \%$ & $20 \%$ \\
& $1.3 \times 10^{4}$ & $12 \%$ & $34 \%$ \\
& $1.1 \times 10^{5}$ & $10 \%$ & $70 \%$ \\
& $1.0 \times 10^{6}$ & $6.8 \%$ & $50 \%$ \\
& $9.0 \times 10^{6}$ & $4.7 \%$ & $23 \%$ \\
& $8.2 \times 10^{7}$ & $3.2 \%$ & $9.1 \%$ \\
& $7.6 \times 10^{8}$ & $3.7 \%$ & $1.7 \%$ \\
& $7.3 \times 10^{9}$ & $2.5 \%$ & $3.8 \%$ \\
& $6.8 \times 10^{10}$ & $2.3 \%$ & $13 \%$ \\
& $6.7 \times 10^{11}$ & $0.63 \%$ & $21 \%$ \\
\hline \hline
\end{tabular}

wall boundary condition is provided). The difference between velocity profiles for cases in Fig. 1(b) can also be explained similarly.

At this point we are ready to examine using LES computational data whether turbulent flows, specifically ZPGTBLs, naturally gravitate towards a log-law or a power-law type scaling. To examine this question quantitatively, the gradients of the velocity profile are computed. The relative difference between the computed velocity gradient and the theoretical one is computed as $\eta=\sqrt{\sum_{i=1, N}\left[\left(\left(G_{L}-G_{T}\right) / G_{T}\right)\right]^{2} / N}$ within the overlap region $\left(z<0.15 \delta_{99}\right)$, where $N$ is the number of points in the overlap region, $G$ denotes gradients, subscripts $L$ denotes LES data ("LESL" for log law assumption and "LES-P" for power law assumption), and $T$ denotes the theoretical value ("log-law" for log law and "GC-law" for power law). These notations are used in Table I.

The difference data are tabulated in Table I and show that both the LES-P and LES-L velocity gradient measurements, on average, are closer to the log-law. The difference between LES-L and the log law monotonically decreases with Reynolds number. For comparison between LES-P and the log law, a general decreasing trend can also be found. When compared with power-law, the difference is larger than that predicted by the theoretical log-law scaling. For high Reynolds number case, the difference between LES and the power law does not show asymptotic behavior. It reaches its nadir at about $R e_{\theta} \sim 10^{9}$, and then rebounds. This phenomenon is valid irrespective of the choice of log-law or power-law assumption in the virtual-wall model and the inflow generation method.

In matching the laws of the inner region and the outer region, a scaled velocity gradient is used. This provides a useful test function for evaluating the overlap profile. ${ }^{9}$ The two functions, $\Xi$ for $\log$ law and $\Gamma$ for power law, are defined as

$$
\Xi \equiv z^{+} \frac{d U^{+}}{d z^{+}}=\frac{1}{\kappa}, \quad \Gamma \equiv \frac{\left(z^{+}+a^{+}\right)}{z^{+}} \cdot \frac{d U^{+}}{d z^{+}}=\gamma .
$$

Results are plotted in Fig. 2. As we emphasize the asymptotic behavior, only seven cases with high Reynolds number are plotted for both LES-L and LES-P. For each case, points far from overlap region $z>0.5 \delta_{99}$ are discarded. It can be found that the number of points in overlap region increases with $\operatorname{Re}_{\theta}$. For the largest $R e$ case in Fig. 2(a), both LES-L and LES-P results agree well with the log-law form, with about 10 points exactly located within the log region. Recalling that for LES-P, the wall model and inflow generation method utilize the GC power-law assumption, it is somewhat surprising that the LES-P law also predicts $\Xi=1 / \kappa$ very well. Although not definitive 

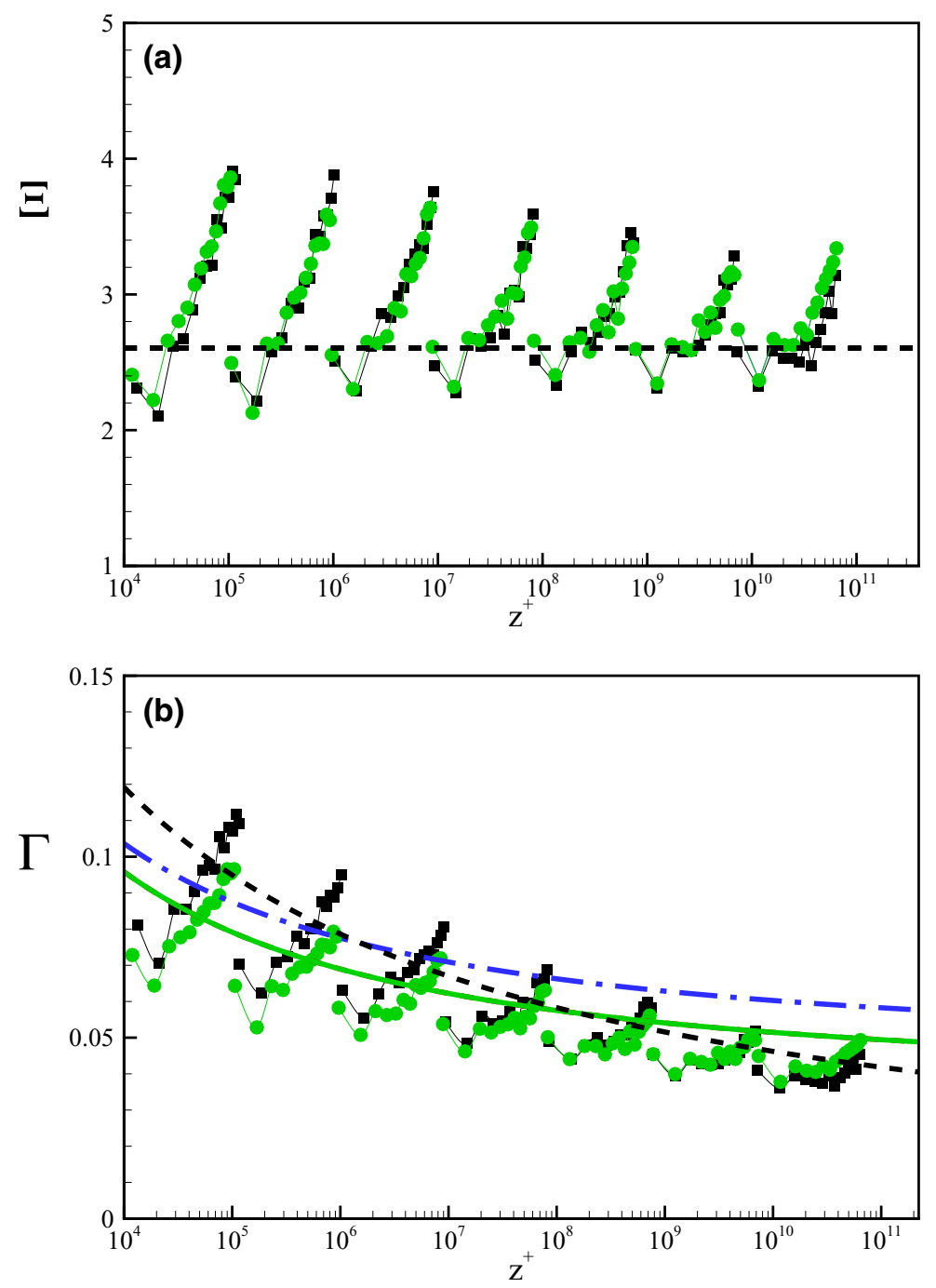

FIG. 2. Scaled velocity gradient in the overlap region. (a) $\Xi$; (b) $\Gamma$. Dashed line: log-law; solid line: GC law; dashed-dotted line: modified GC law parameters; ${ }^{9}$ squares: LES with log-law; circles: LES with power-law. Every curve with symbols corresponds to different $R e_{\theta}$ ranging from $10^{6}$ to $10^{11}$ in Table I.

proof, this numerical evidence indicates that high-Reynolds number wall-bounded turbulent flows tend to preferentially obey the log-law scaling. For $\Gamma$, the numerical results again do not show much difference. However, $\Gamma$ from numerical results is smaller than the GC law exponent $\gamma$. This leads us to explore a modification of $\gamma$ in the GC law proposed by Monkewitz et al., ${ }^{9}$ aimed at providing a better explanation of experiments for medium Reynolds numbers. This version of $\gamma$ is also plotted in Fig. 2(b). However, we conclude that the Monkewitz modification also shows significant deviations at high Reynolds numbers and the comparison with LES results is worse than $\gamma$ from the original GC law.

Conclusion: Our LES methodology is a viable approach (one based on computations as opposed to experiments or theoretical scaling arguments) to answer the log-law versus power-law debate. The computed velocity profiles, velocity gradients, and scaled velocity gradients show that LES results are quantitatively in better agreement with the log law than the GC power law. Intuitively, we expect that results from LES with the log-law assumption in the virtual wall model (LES-L) to be closer to the log-law behavior of the mean streamwise velocity. But the agreement between LES-P (with only power law assumption) results and the log-law $\kappa$ constant is strong evidence that the log law is robust at least at high Reynolds numbers. We note that the extreme Reynolds number values 
$\left(R e_{\theta} \sim 10^{11}\right)$ in present LES cannot be attained by experiments or DNS in the foreseeable future. However, because the subgrid model and wall model are inherent components of the LES, it is difficult to evaluate and impossible to eliminate the influence of models on the simulation results. Moreover, the recycling method used for inflow boundary condition adds additional numerical uncertainty. For these reasons, while our results are convincing about the validity of the log-law, our results are not a conclusive proof. Finally, we assumed a smooth wall ZPGTBL. Influence of surface roughness, other realistic flows (e.g., atmospheric TBL) at extreme Reynolds numbers, and scaling behavior for other types of wall-bounded turbulent flows are still open questions. To address these questions, rational LES frameworks such as that used in the current work are an inevitable and important tool for the whole turbulence community to probe other physics questions in high Reynolds number wall-bounded turbulence.

\section{ACKNOWLEDGMENTS}

Wan Cheng was supported by CCRC, KAUST. We gratefully acknowledge computer time on Shaheen, the IBM Blue Gene P supercomputer at KAUST.

${ }^{1}$ G. I. Barenblatt, "Scaling laws for fully developed turbulent shear flows. Part 1. Basic hypotheses and analysis," J. Fluid Mech. 248, 513-520 (1993).

${ }^{2}$ G. I. Barenblatt, "Characteristic length scale of the intermediate structure in zero-pressure-gradient boundary layer flow," Proc. Natl. Acad. Sci. U.S.A. 97, 3799-3802 (2000).

${ }^{3}$ W. George and L. Castillo, "Zero-pressure-gradient turbulent boundary layer," Appl. Mech. Rev. 50, 689 (1997).

${ }^{4}$ W. George, "Recent advancements towards the understanding of turbulent boundary layer," AIAA J. 44, 2435-2449 (2006).

${ }^{5}$ M. V. Zagarola and A. J. Smits, "Mean-flow scaling of turbulent pipe flow," J. Fluid Mech. 373, 33-79 (1998).

${ }^{6} \mathrm{~N}$. Afzal, "Power law and log law velocity profiles in fully developed turbulent pipe flow: Equivalent relations at large Reynolds numbers," Acta Mech. 151, 171-183 (2001).

${ }^{7}$ B. J. Mckeon, J. Li, W. Jiang, J. F. Morrison, and A. J. Smits, "Further observations on the mean velocity distribution in fully developed pipe flow," J. Fluid Mech. 501, 135-147 (2004).

${ }^{8}$ M. Buschmann and M. Gad-el-Hak, "Debate concerning the mean-velocity profile of a turbulent boundary layer," AIAA J. 41, 565-572 (2003).

${ }^{9}$ P. Monkewitz, K. A. Chauhan, and H. Nagib, "Comparison of mean flow similarity laws in zero pressure gradient turbulent boundary layers," Phys. Fluids 20, 105102 (2008).

${ }^{10}$ I. Marusic, B. J. McKeon, P. A. Monkewitz, H. M. Nagib, and A. J. Smits, "Wall-bounded turbulent flows at high Reynolds numbers: Recent advances and key issues," Phys. Fluids 22, 065103 (2010)

${ }^{11}$ M. Hultmark, M. Vallikivi, S. Bailey, and A. Smits, "Turbulent pipe flow at extreme reynolds numbers," Phys. Rev. Lett. 108, 094501 (2012).

${ }^{12}$ I. Marusic, J. P. Monty, M. Hultmark, and A. J. Smits, “On the logarithmic region in wall turbulence,” J. Fluid Mech. 716, R3 (2013).

${ }^{13} \mathrm{M}$. Inoue and D. I. Pullin, "Large-eddy simulation of the zero pressure gradient turbulent boundary layer up to $R e_{\theta}$ $=o\left(10^{12}\right)$, , J. Fluid Mech. 686, 507-533 (2011).

${ }^{14}$ D. Chung and D. I. Pullin, "Large-eddy simulation and wall modelling of turbulent channel flow," J. Fluid Mech. 631, 281-309 (2009).

${ }^{15}$ K. A. Chauhan, P. A. Monkewitz, and H. M. Nagib, "Criteria for assessing experiments in zero pressure gradient boundary layers," Fluid Dyn. Res. 41, 021404 (2009).

${ }^{16}$ M. Wosnik, L. Castillo, and W. K. George, “A theory for turbulent pipe and channel flows,” J. Fluid Mech. 421, 115-145 (2000).

${ }^{17}$ T. S. Lund, X. Wu, and K. D. Squires, "Generation of turbulent inflow data for spatially developing boundary layer simulations," J. Comput. Phys. 140, 233-258 (1998).

${ }^{18}$ G. Araya, K. E. Jansen, and L. Castillo, "Inlet condition generation for spatially developing turbulent boundary layer via multiscale similarity," J. Turbul. 10(36), 1-33 (2009).

${ }^{19}$ W. Cheng and R. Samtaney, "A high-resolution code for Large Eddy Simulation of incompressible turbulent boundary layer flows," Comput. Fluids 92, 82-92 (2014).

${ }^{20}$ H. M. Nagib, K. A. Chauhan, and P. A. Monkewitz, "Approach to an asymptotic state for zero pressure gradient turbulent boundary layers," Philos. Trans. R. Soc. A 365, 755-770 (2007). 